\title{
Relapsing Polychondritis Associated with Myelodysplastic Syndrome
}

\author{
Mahfoudhi Madiha*, Mamlouk Habiba, Lahiani Rym, Turki Sami, Kheder Adel \\ Department of Internal Medicine A, Charles Nicolle Hospital, Tunis, Tunisia \\ Email: ${ }^{*}$ madiha_mahfoudhi@yahoo.fr
}

Received 12 February 2015; accepted 3 March 2015; published 10 March 2015

Copyright (C) 2015 by authors and Scientific Research Publishing Inc.

This work is licensed under the Creative Commons Attribution International License (CC BY). http://creativecommons.org/licenses/by/4.0/

(c) () Open Access

\begin{abstract}
A 62-year-old man had arthritis and an infiltrated purpuric eruption of the legs. He presented then a cyanosis and aches of the left ear's pinna with a voice disturbance and an inflammatory aspect of the nose. He had an anemia and a thrombopenia. The infectious and auto-immune research was negative. The laryngo-tracheal scan revealed calcifications of the larynx, the trachea and the initial portion of bronchi. Moreover, the biopsy from ear's pinna cartilage confirmed the diagnosis of chondritis and peri-chondritis. He was thus considered to have the association with relapsing polychondritis and a myelodysplastic syndrome. The patient was treated with corticosteroids and iterative transfusions. The articular and skinny patient's symptoms completely resolved. The anemia and the thrombopenia were partially improved. The relapsing polychondritis can be considered as paraneoplastic complication in the context of a myelodysplastic syndrome or as a fortuitous association. The mechanism of such an association remains unknown.
\end{abstract}

\section{Keywords}

Relapsing Polychodritis, Biopsy, Myelodysplastic Syndrome, Prognosis

\section{Introduction}

The relapsing polychondritis [1] is an acquired disease of unknown etiology, chronic course, and tendency to recur. It is characterized by inflammation and degeneration of cartilage and can result in deformities such as floppy ear and saddle nose. Loss of cartilage in the respiratory tract can lead to respiratory obstruction. It's a rare inflammatory disease which can implicate organs rich of proteoglycan as the eyes, the heart, the vessels and the kidneys [2].

The case highlights the association between relapsing polychondritis and myelodysplastic syndrome. This as-

\footnotetext{
${ }^{*}$ Corresponding author.
} 
sociation is rare, and the mechanism remains unknown, but it has significant clinical implication leading to severe features threatening the vital prognosis.

\section{Case Presentation}

A 62-year-old man was referred to internal medicine department with symptoms of recurrent arthritis and leg's purpura since seven months. The patient had a significant medical history. He was followed up for a type 2 diabetis and a hypertension for 12 years. He was treated for pulmonary tuberculosis five years ago. No new treatment was introduced recently.

The clinical examination revealed arthritis of proximal and distal interphalangeal joint of fingers, an infiltrated purpuric eruption of the legs.

He presented during his hospitalization cyanosis and aches of the left ear's pinna with a voice disturbance, an inflammatory aspect of the nose and an ache at the pressure of nasal cartilage.

The ORL examination confirmed a decrease of hearing. The ophthalmological control revealed a diabetic retinopathy.

Suspicion for an underlying autoimmune disease or a systemic vasculitis was raised.

He had an anemia (Hemoglobin: $7 \mathrm{~g} / \mathrm{dl}$ ), associated with a thrombopenia (Platelets: 70,000/mm3). Serology showed elevated erythrocyte sedimentation rate, normal complement levels, negative antinuclear antibody antiphospholipid and antidouble-stranded DNA, Cytoplasmic Antineutrophil Antibodies (ANCA), rheumatoid factor and anticyclic citrullinated peptide antibody. His liver and kidney functions were normal.

The infectious investigations were negative.

The laryngo-tracheal scan (Figure 1) concluded to calcifications of the larynx, the trachea and the initial portion of bronchi.

The cardiac echography as well as all the conventional radiographs was normal.

A bone marrow biopsy confirmed a myelodysplastic syndrome subtype refractory anaemia.

A skin biopsy of purpuric lesions revealed a leucocytoclasic vasculitis.

The biopsy from ear's pinna cartilage confirmed the diagnosis of chondritis and peri-chondritis.

He was thus considered to have the association of relapsing polychondritis and a myelodysplastic syndrome.

The patient was treated with a prolonged corticosteroids therpy. And he had received several transfusions.

The articular and skinny patient's symptoms completely resolved. The anemia and the thrombopenia were partially improved.

He was followed by internal medicine and hematology department for this association.

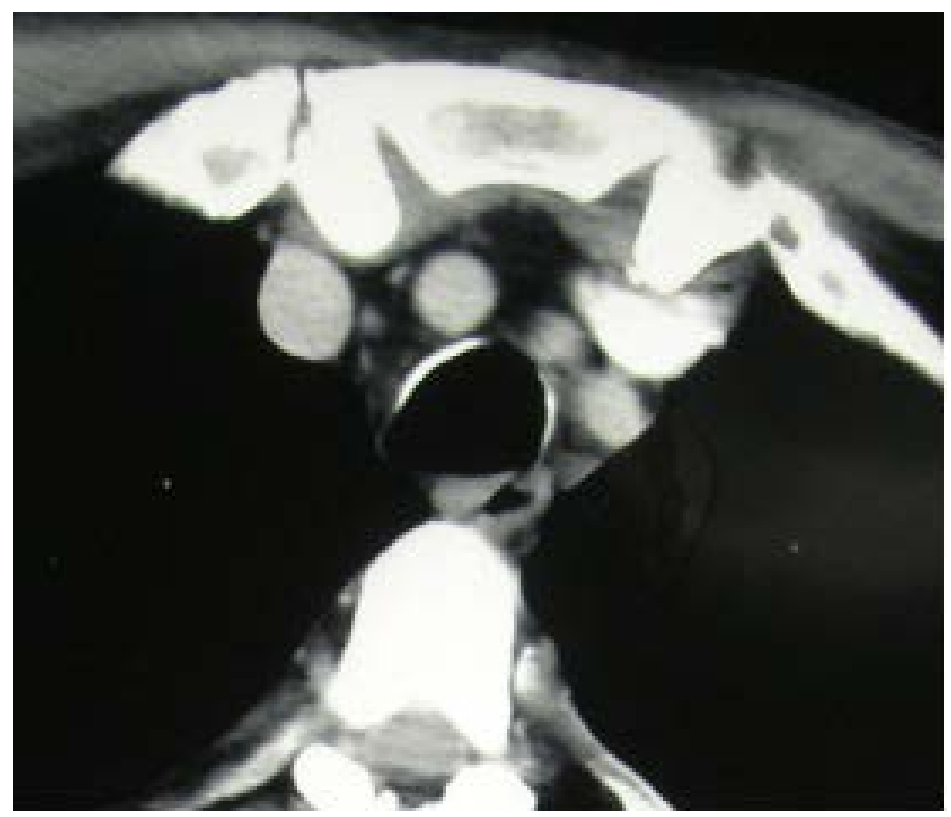

Figure 1. Laryngo-tracheal scan: calcifications of the trachea. 


\section{Discussion}

The relapsing polychondritis is a progressive cartilage inflammation, leading to its destruction in ears, nose, larynx trachea and joints [1].

It’s a rare disease including visceral manifestations concerning eyes, heart, vessels and kidney [2].

Three or more clinical signs must be present:

1) Recurrent chondritis of both auricles;

2) Non-erosive inflammatory polyarthritis;

3) Nasal chondritis;

4) Ocular inflammation;

5) Respiratory tract chondritis;

6) Cochlear and/or vestibular dysfunction.

The association of one or more signs among the above mentioned signs with histologic confirmation is in favour of the relapsing polychondritis diagnosis.

Chondritis in two or more separate sites and response to steroids or immunosuppression is also suggestive of this pathology.

The diagnosis of relapsing polychondritis was confirmed in our patient by the association of four signs (inflammation of nasal, auricular and laryngo-tracheal cartilage and arthritis).

The histological study of cartilage biopsy confirmed the chondritis in our patient. Nevertheless, the biopsy is not indispensable for the diagnosis.

The first symptoms are ache and tumefaction due to ear's chondritis, then nasal signs and a conduction hearing loss [3].

The main manifestations of this disease are articular and muscular signs, ocular symptoms (episcleritis, scleritis iritis, retinopathy...), ORL signs (cough, stridor, inspiratory dyspnea...), pulmunory ones (restrictif syndrome), renal ones (hematuria, proteinuria...), skinny ones (purpura, nodules...), cardiovascular ones (aortic lesion...) [4].

The treatment is based on corticosteroids and immunosuppressive molecules [5]. A clinical and biological improvement was signed in our patient after receiving only corticosteroids. Hematologic disturbances were refractory to this treatment and required several transfusions.

The relapsing polychondritis can threaten the vital prognosis in case of a diagnostic delay and treatment and in particular localization like laryngo-tracheal one. Our patient had not presented this complication.

This disease can be associated to vasculitis, auto-immune diseases, spondylarthropathies, hematologic diseases and other systemic diseases [1].

The association with a myelodysplastic syndrome (erythroid hypoplasia/aplasia) is frequent.

This association is considered significant and is reported in many series. The etiopathogeny of this association is not established. The occurrence of these two affections is usually concomitant [5].

The relapsing polychondritis is present in less than $1 \%$ of myelodysplastic syndromes, while $25 \%$ of relapsing polychondritis are associated with myelodysplastic syndrome [5]-[8].

The prognosis of this association is worsened in case of refractory myelodysplastic syndrome.

The association of a systemic disease and a myelodysplastic syndrome may not be a coincidence. 14 cases of myelodysplatic syndrome having this association were reported, and 2 of them presented relapsing polychondritis [6].

The relapsing polychondritis can be considered as paraneoplastic complication in the context of a myelodysplastic syndrome, or a fortuitous association. The mechanism of such an association remains unknown [1] [7] [8]. There would be compatibility between auto-antibodies produced in myelodysplastic syndromes and various cartilaginous structures; thus leading to classical lesionfs of relapsing polychondritis.

\section{Conclusion}

The clinical presentation of the relapsing polychondritis is heterogeneous. The association of the myelodysplastic syndrome may induce a lateness of the diagnostic delay. And the cartilage biopsy has a high interest in the diagnostic confirmation.

\section{Conflict of Interest}

The authors declare that there is no conflict of interests regarding the publication of this paper. 


\section{References}

[1] Calistru, A.M., Lisboa, C. and Azevedo, F. (2011) Paraneoplastic Relapsing Polychondritis and Sweet Syndrome Coexisting in a Patient with Myelodysplasia. Indian Journal of Dermatology, Venereology and Leprology, 77, 730. http://dx.doi.org/10.4103/0378-6323.86505

[2] Coha, B., Fustar-Preradovic, L., Sekelj, S. and Sekelj, A. (2007) Total Hearing Loss and Blindness Caused by Relapsing Polychondritis and Myelodysplastic Syndrome. European Archives of Oto-Rhino-Laryngology, 264, 1517-1519. http://dx.doi.org/10.1007/s00405-007-0387-9

[3] Banerjee, S.S., Morris, D.P., Rothera, M.P. and Routledge, R.C. (2001) Relapsing Polychondritis Associated with Monoclonal Gammopathy in a Patient with Myelodysplastic Syndrome. The Journal of Laryngology \& Otology, 115, 482-484.http://dx.doi.org/10.1258/0022215011907983

[4] Loeffler, K.U. and McLean, I.W. (2000) Bilateral Necrotizing Scleritis and Blindness in the Myelodysplastic Syndrome Presumably Due to Relapsing Polychondritis. Acta Ophthalmologica Scandinavica, 78, 228-231. http://dx.doi.org/10.1034/j.1600-0420.2000.078002228.x

[5] Myers, B., Gould, J. and Dolan, G. (2000) Relapsing Polychondritis and Myelodysplasia: A Report of Two Cases and Review of the Current Literature. Clinical \& Laboratory Haematology, 22, 45-48. http://dx.doi.org/10.1046/j.1365-2257.2000.00268.x

[6] Roy-Peaud, F., Paccalin, M., Le Moal, G., Landron, C., Juhel, L., Roblot, P., et al. (2003) Association of Systemic Diseases and Myelodysplastic Syndromes. A Retrospective Study of 14 Cases. La Presse Médicale, 32, 538-543.

[7] Chopra, R., Chaudhary, N. and Kay, J. (2013) Relapsing Polychondritis. Rheumatic Disease Clinics of North America, 39, 263-276.http://dx.doi.org/10.1016/j.rdc.2013.03.002

[8] Washio, K., Oka, M., Ohno, K., Shimizu, H., Kawano, S., Kunisada, M., et al. (2012) Case of Recurrent Sweet’s Syndrome in a Patient with Relapsing Polychondritis and Myelodysplastic Syndrome. The Journal of Dermatology, 39, 731-733. http://dx.doi.org/10.1111/j.1346-8138.2011.01403.x 